\title{
ECOLOGÍA Y ESTRUCTURA POBLACIONAL DEL ENDEMISMO CUBANO TETRAMICRA MALPIGHIARUM (ORCHIDACEAE), EN EL PARQUE NACIONAL DESEMBARCO DEL GRANMA, CUBA
}

\author{
Alfredo García-González ${ }^{1,5}$, Frander B. Riverón-Giró, \\ Ixchel S. GonzÁlez-Ramírez ${ }^{2}$, Raisa Y. Escalona Domenech ${ }^{1}$, \\ Yamileth Hernández Montero ${ }^{3}$ \& Ernesto Palacio Verdecia ${ }^{4}$ \\ ${ }^{1}$ El Colegio de la Frontera Sur (ECOSUR), Unidad Tapachula. Carretera Antiguo Aeropuerto, km 2.5, \\ Apartado Postal 36, Tapachula, Chiapas, México. C.P. 30700 \\ ${ }^{2}$ Universidad Nacional Autónoma de México (UNAM), Instituto de Geología, México D.F., México \\ ${ }^{3}$ Jardín Botánico de Holguín. Holguín, Cuba \\ ${ }^{4}$ Parque Nacional Desembarco del Granma, Granma, Cuba \\ ${ }^{5}$ Autor para correspondencia: alfredmx22@gmail.com
}

Resumen. En Cuba se reconocen aproximadamente 315 especies para la familia Orchidaceae, de las cuales son endémicas alrededor de $32 \%$. Durante 2012 y 2013, se estudió una población del endemismo cubano Tetramicra malpighiarum (Orchidaceae), en el Parque Nacional Desembarco del Granma, Cuba. Se estudió la abundancia de la especie, clases de vida de los individuos (inmaduros y adultos), forófitos sobre los que crecían, micrositios que ocupaban en los mismos (tronco, ramas o ramillas), si los individuos estaban solitarios o agregados y orientación cardinal que tenían sobre los forófitos. Se encontraron 254 individuos de T. malpighiarum, distribuidos en 20 forófitos. Por primera vez se localizaron individuos de T. malpighiarum ocupando otras cinco especies de forófitos, además de Malpighia incana (Malpighiaceae) y Erythroxylum havanense (Erythroxylaceae). Fueron adultos $69.3 \%$ de los individuos y en las ramillas crecía $93.7 \%$ de la población. Es similar el número de individuos de T. malpighiarum creciendo agregados y creciendo solitarios. La población muestra una pequeña tendencia a crecer preferentemente con orientaciones sur y noroeste. Este trabajo es una primera aproximación a la ecología poblacional de T. malpighiarum, pero es fundamental realizar estudios genéticos y demográficos a largo plazo, que permitan monitorear el comportamiento poblacional y reproductivo de este endémico cubano, y averiguar los niveles de endogamia y variabilidad genética de los individuos, para conocer su posible comportamiento y permanencia en el futuro.

ABSTRACT. In Cuba, there are 315 species of Orchidaceae family, approximately $32 \%$ of these species are endemic. During 2012 and 2013 an endemic population of Tetramicra malpighiarum (Orchidaceae) was studied at the Desembarco del Granma National Park, Cuba. We determined the abundance of this species, life classes (immature and adult), phorophytes inhabited, vertical distribution (trunk, branches or twigs), solitary or aggregated growing, and its cardinal orientation on phorophytes. We found 254 individuals of T. malpighiarum distributed in 20 phorophytes. For the first time, were found individuals of T. malpighiarum grew on other five species of phorophytes, besides of Malpighia incana (Malpighiaceae) and Erythroxylum havanense (Erythroxylaceae). Of the total of individuals recorded, $69.3 \%$ were adult and $93.7 \%$ were growing on the twigs. The number of solitary individual and aggregated individuals was similar. The population shows a slight tendency to grow toward south and northwest orientations. This is the first study to describe the population ecology of T. malpighiarum, but it is recommended to conduct long-term genetic and demographic studies. These future studies should be explored the reproductive system (e.g., level of endogamy) and population genetic of this species endemic of Cuba in order to evaluate its permanency in the future.

Palabras clave: clases de vida, distribución vertical, forófito, Malpighia incana, orientación cardinal 
Introducción. Cuba constituye la cuarta isla con mayor diversidad de plantas del mundo (Whittaker \& Fernández-Palacios 2007), y la primera en cuanto al número de especies por $\mathrm{km}^{2}$ (González-Torres et al. 2013). Una de las características más distintivas de la flora cubana es su alto endemismo; $53 \%$ de la flora nativa son especies endémicas (Berazaín et al. 2005). No obstante esta gran riqueza florística, sobre la flora del país pesan numerosas amenazas, tanto de origen natural como antrópico, lo que hace imprescindible elevar el conocimiento que se tiene de las especies de plantas cubanas y de su situación de conservación (González-Torres et al. 2013).

Particularmente para la familia Orchidaceae, en Cuba se reconocen aproximadamente 315 especies (Ackerman 2014a), de las cuales $71 \%$ son epífitas (Díaz 1999), y el endemismo es de alrededor de $32 \%$ (Ackerman 2014a). Una de las especies de orquídeas epífitas endémicas de Cuba es Tetramicra malpighiarum J.A. Hern. \& M.A. Díaz (Fig. 1A - 1B)
(Hernández \& Díaz 2000, Llamacho \& Larramendi 2005, Acevedo-Rodríguez \& Strong 2012, Ackerman 2014b). De este taxón, catalogado como en peligro crítico (CR; Berazaín et al. 2005), solo se conocen dos poblaciones, de pocas plantas cada una y gran especificidad en la elección de forófito (Hernández \& Díaz 2000, Llamacho \& Larramendi 2005), creciendo fundamentalmente sobre Malpighia incana Mill. (Malpighiaceae; Hernández \& Díaz 2000, Llamacho \& Larramendi 2005, García-González et al. 2013). Habita en la costa norte de la provincia Ciego de Ávila y en la costa sur de la porción oriental de la isla, y su población más importante se localiza al oriente de Cuba, en El Guafe, Parque Nacional Desembarco del Granma (PNDG), provincia Granma (Hernández \& Díaz 2000, Llamacho \& Larramendi 2005, GarcíaGonzález et al. 2013).

Teniendo en cuenta que T. malpighiarum es una especie de orquídea con una distribución muy limitada, poblaciones muy reducidas y gran especificidad en
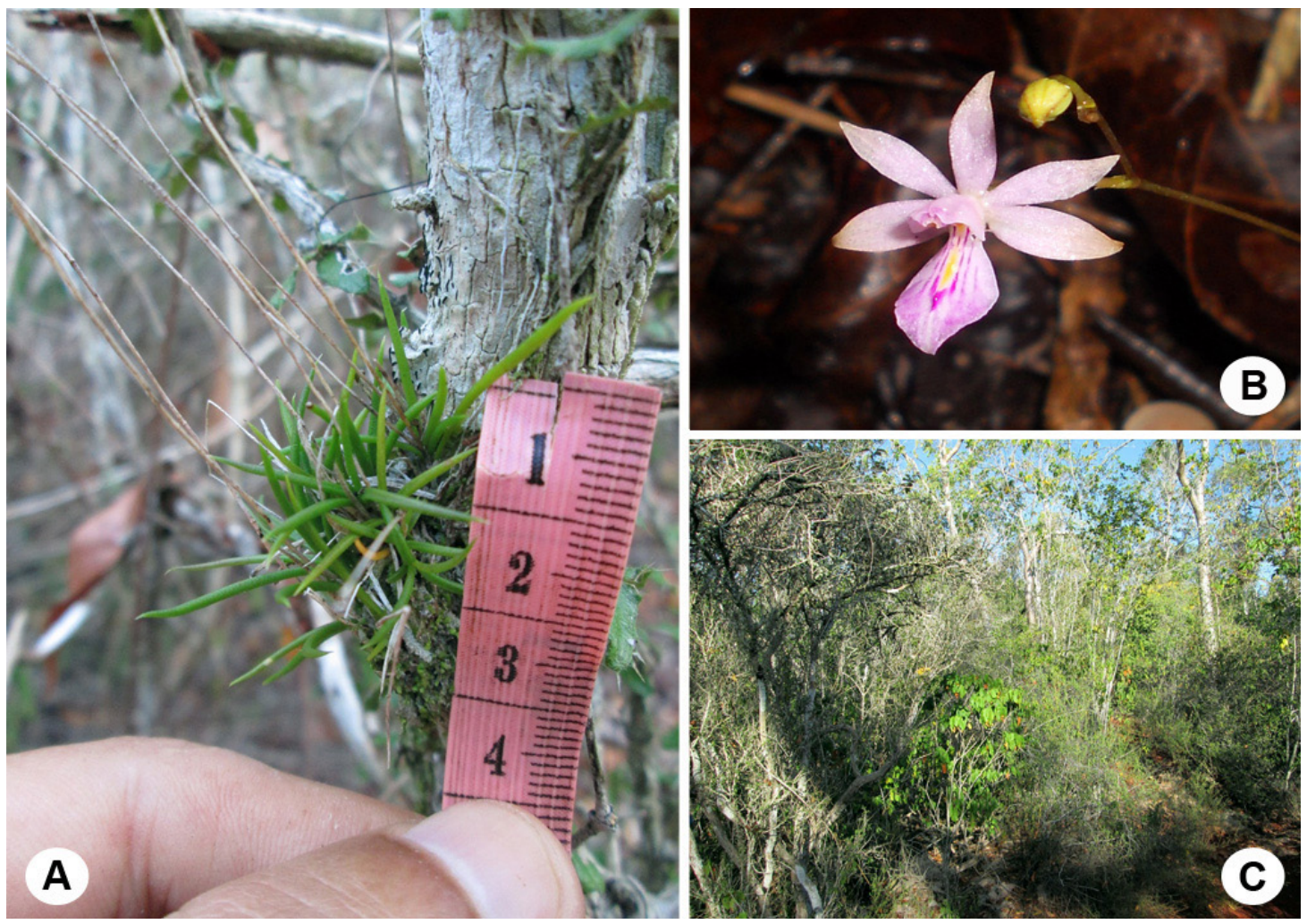

Figura 1. Tetramicra malpighiarum (Orchidaceae) en el Parque Nacional Desembarco del Granma, Cuba. A: Planta en su hábitat, B: Flor, C: Bosque transicional entre matorral xeromorfo costero y bosque semideciduo (hábitat). 
la elección de sustrato (Hernández \& Díaz 2000, Llamacho \& Larramendi 2005). Se procedió a estudiar su abundancia y estructura poblacional, como una primera aproximación al conocimiento de su ecología poblacional y como una forma de contribuir a aumentar el escaso conocimiento que se tiene sobre esta especie, todo lo cual redundará en mejores acciones de conservación y manejo para garantizar la permanencia de este endémico cubano.

Materiales y Métodos. Caracterización del sitio -. El estudio se desarrolló durante 2012 y 2013, en el área donde se localiza la población tipo de T. malpighiarum (Hernández \& Díaz 2000), una zona de transición entre matorral xeromorfo costero y bosque semideciduo (Fig. 1C; García-González et al. 2013), en los $19^{\circ} 51^{\prime}$ $186^{\prime \prime} \mathrm{N} ; 77^{\circ} 42^{\prime} 690^{\prime \prime} \mathrm{W}$, en El Guafe, PNDG, Cuba. En esta área el promedio anual de precipitaciones oscila entre 800 y $1200 \mathrm{~mm}$, y la temperatura entre 30.5 y $22.9^{\circ} \mathrm{C}$ (Palacio et al. 2012).

Toma de datos -. Con el objetivo de localizar nuevos individuos y forófitos de T. malpighiarum, se desarrolló un diseño radial cuyo centro fue el núcleo poblacional de la especie, reportado por Hernández \& Díaz (2000). A partir de ese centro, se trazaron ocho transectos de $800 \mathrm{~m}^{2}$ cada uno $(10 \times 80 \mathrm{~m} ; 0.08 \mathrm{ha})$, dentro de los cuales se contaron los individuos de T. malpighiarum. Los esfuerzos de muestreo se concentraron en esta área, porque se consideró que era más factible encontrar nuevas plantas de T. malpighiarum en sitios cercanos a las plantas madres (Machon et al. 2003, Trapnell et al. 2004, Jersáková \& Malinová 2007, Winkler et al. 2009, Chung et al. 2011).

Caracterización de los forofitos de T. malpighiarum. Se identificaron taxonómicamente todos los forófitos (MBG 2015a), se midió su diámetro a la altura del pecho (DAP; cm), y se estimó su altura (m) siguiendo la metodología propuesta por García-González et al. (2011). En este trabajo el término forófito sólo se utiliza para los arbustos y árboles sobre los que crecía T. malpighiarum (García-González \& Pérez 2011).

Preferencias de establecimiento de T. malpighiarum-. Con el objetivo de conocer si existía una tendencia en el establecimiento de T. malpighiarum en los forófitos, se estimaron las alturas a las que se ubicaban los individuos en los arbustos y árboles, y se midieron los diámetros de las secciones de los tronco o de las ramas donde crecían. Además, se registró la distribución vertical de cada individuo dentro de su forófito, siguiendo la zonación vertical propuesta por GarcíaGonzález \& Riverón-Giró (2014) para árboles de naranjo (Citrus sinensis (L.) Osbeck; Rutaceae). Es importante señalar que por un error de los autores, en García-González \& Riverón-Giró (2014) aparece que el parámetro utilizado para clasificar los micrositios "ramas y ramillas" fue la circunferencia, cuando en realidad se utilizó el diámetro (comentario de los autores; García-González A. \& Riverón-Giró F.B.). Se empleó esta clasificación porque es la que más se ajusta a las características ecológicas de los micrositios presentes en los arbustos y árboles encontrados en el área estudiada.

Clases de vida de los individuos de T. malpighiarum, orientaciones cardinales que tienen en los forófitos, y estado de agregación en el que crecen -. Siguiendo las metodologías y criterios establecidos por GarcíaGonzález \& Riverón-Giró (2014), todos los individuos de T. malpighiarum se incluyeron en dos clases de vida (adultos o inmaduros), y se registraron las orientaciones cardinales en las que se encontraban ubicados en los forófitos. El estado de agregación de los individuos se determinó siguiendo los criterios utilizados por González et al. (2007) y Mújica (2007) para Broughtonia cubensis (Lindl.) Cogn. No obstante, teniendo en cuenta el menor tamaño promedio de $T$. malpighiarum, se desarrolló una versión específica para esta especie.

Los individuos de T. malpighiarum se dividieron en dos grupos o tratamientos:

- Individuos agregados: Aquellos que están a una distancia de $5 \mathrm{~cm}$ o menos de otro individuo de T. malpighiarum o de otras especies de epífitas vasculares.

- Individuos solitarios: Aquéllos que están a una distancia mayor de $5 \mathrm{~cm}$ de otro individuo de T. malpighiarum, de otras especies de epífitas vasculares o habitando en solitario en un forófito.

Se consideró la distancia mínima de $5 \mathrm{~cm}$ para determinar agregación, teniendo en cuenta el pequeño tamaño de T. malpighiarum (plantas sin pseudobulbos 
TABLA 1. Especies de forófitos sobre los que crece Tetramicra malpighiarum (Orchidaceae), en el Parque Nacional Desembarco del Granma, Cuba; número de forófitos registrados por especie, y número de individuos de T. malpighiarum que se encontraron ocupando cada especie de forófito.

\begin{tabular}{l|l|c|c}
\hline Especies de forófitos & Familia & $\begin{array}{c}\text { No. de forófitos } \\
\text { registrados }\end{array}$ & $\begin{array}{c}\text { No. de individuos } \\
\text { de T. malpighiarum }\end{array}$ \\
\hline Malpighia incana & Malpighiaceae & 14 & 217 \\
\hline Erythroxylum havanense & Erythroxylaceae & 1 & 2 \\
\hline Eugenia anthacanthoides & Myrtaceae & 1 & 24 \\
\hline Guettarda elliptica & Rubiaceae & 1 & 3 \\
\hline Plumeria obtusa & Apocynaceae & 1 & 2 \\
\hline Randia aculeata & Rubiaceae & 1 & 5 \\
\hline Stigmaphyllon sagreanum & Malpighiaceae & 1 & 1 \\
\hline Total & ---- & 20 & 254 \\
\hline
\end{tabular}

y 4-6 cm de alto; Llamacho \& Larramendi 2005). A pesar de que las raíces de los individuos se entrecruzan a mayor distancia, fue imposible incluir este parámetro para delimitar la distancia de agregación. Generalmente las raíces de estas plantas no son visibles fácilmente en toda su extensión, son muy delgadas $(0.5-1 \mathrm{~mm}$ de diámetro), de color blanco a gris (Ackerman 2014b), y se extienden por dentro de las grietas de la corteza de los forófitos y por debajo de los líquenes que la cubren.

Análisis estadísticos - . Las medidas de tendencia central que se expresan en el texto son promedios, mientras que las medidas de variación son errores estándar. Se realizó una prueba de $\mathrm{x}^{2}$ con tabla de contingencia para evaluar el efecto del estadio de vida de los individuos de T. malpighiarum sobre su estado de agregación. Así mismo, se comparó la frecuencia de establecimiento de los individuos en distintas categorías de altura y grosor de las ramas, con un modelo de distribución aleatorio generado con una tabla de contingencia. Finalmente, se realizó una prueba de Uniformidad Circular de Rayleigh, en el caso de la variable orientación cardinal de los individuos en los forófitos, utilizó el programa Oriana (Versión 1.01).

Resultados Caracterización de la población de T. malpighiarum -. Se encontraron 254 individuos de T. malpighiarum (Tabla 1), en $\operatorname{los} 6400 \mathrm{~m}^{2}$ (0.64 ha) muestreados. Sin embargo, todos los individuos se concentraron en un área de tan solo $400 \mathrm{~m}^{2}$, en los primeros metros de los transectos, en y alrededor del núcleo poblacional original de la especie. La población está formada principalmente por individuos adultos (69.3\% de la población; 176 individuos; Fig. 2).

Se encontraron creciendo solitarios $51.18 \%$ de los individuos (130 individuos), y el resto crecían agregados (Fig. 2), principalmente con otras orquídeas de su misma especie (115 individuos de $T$. malpighiarum agregados entre ellos). No obstante, se registró agregación de $T$. malpighiarum con otras especies de epífitas vasculares como, Tillandsia fasciculata var. clavispica Mez (Bromeliaceae; 6 individuos de T. malpighiarum agregados con esta especie), Tolumnia guibertiana (A. Rich.) Braem (Orchidaceae; 2 individuos de T. malpighiarum

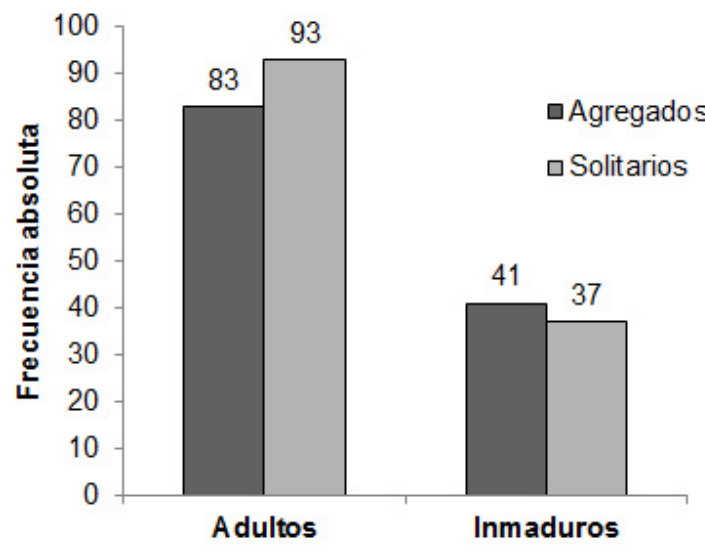

FIgURA 2. Frecuencia absoluta de individuos de Tetramicra malpighiarum (Orchidaceae) creciendo solitarios y agregados, según su estadio de vida, en el Parque Nacional Desembarco del Granma, Cuba. 
agregados con esta especie) y Broughtonia lindenii (Lindl.) Dressler (Orchidaceae: 1 individuo de $T$. malpighiarum agregado con esta especie). También, se observó que la frecuencia de agregación no depende del estadio de vida de los individuos de $T$. malpighiarum $\left(\chi^{2}=0.63, \mathrm{p}=0.42\right)$. Otras especies de epífitas vasculares y hemiepífitas, que aunque no se encontraron en agregación con T. malpighiarum, se observaron creciendo simpátricamente con esta orquídea, fueron: Encyclia phoenicea (Lindl.) Newmann, Vanilla sp. (Orchidaceae), Tillandsia balbisiana Schult. \& Schult.f., T. bulbosa Hook., T. flexuosa Sw., T. recurvata (L.) L., T. usneoides (L.) L., T. utriculata L. (Bromeliaceae) y Selenicereus grandiflorus (L.) Britton \& Rose (Cactaceae).

Caracterización de los forófitos de T. malpighiarum -. Se encontraron 20 forófitos, pertenecientes a siete especies y cinco familias botánicas (Tabla 1). La especie de forófito más importante fue $M$. incana $(70 \%$ de todos los forófitos; 14 plantas), y sobres esta especie crecía la mayor parte de la población de $T$. malpighiarum (85.43\% de la población; 217 individuos; Tabla 1). Los forófitos de la especie $M$. incana tuvieron en promedio $15.5 \pm 6.77$ individuos de T. malpighiarum, mientras que el resto de los forófitos en conjunto promediaron $6.16 \pm 2.52$ individuos por forófito. La altura promedio de los forófitos en general fue de $199.1 \pm 32.4 \mathrm{~cm}$ y un diámetro de $2.73 \pm 0.62 \mathrm{~cm}$.

Preferencias de establecimiento de T. malpighiarum Los individuos de T. malpighiarum crecían fundamentalmente sobre ramillas $(93.7 \%$ de la población; 238 individuos), únicamente $6.3 \%$ de la población (16 individuos) se encontró creciendo en troncos, y no se localizaron individuos creciendo en ramas. Los individuos se encontraron a alturas que iban desde 10 hasta $220 \mathrm{~cm}$ (Fig. 3A), y en troncos y ramillas cuyos diámetros iban de 0.22 a $12.48 \mathrm{~cm}$ (Fig. 3B). Sin embargo, la distribución de los individuos no fue aleatoria, ni en el grosor de las ramas $\left(\chi^{2}=949.87\right.$, $\mathrm{p}<0.0001)$, ni en la altura de las mismas $\left(\chi^{2}=199.63, \mathrm{p}<\right.$ $0.0001)$. Los individuos se establecían principalmente en troncos y ramillas que se encontraban por debajo de $80 \mathrm{~cm}$ de altura (Fig. 3A), y que tuvieran menos de $2.22 \mathrm{~cm}$ de grosor (Fig. 3B).

Por otra parte, se encontraron individuos de $T$. malpighiarum creciendo hacia las ocho orientaciones
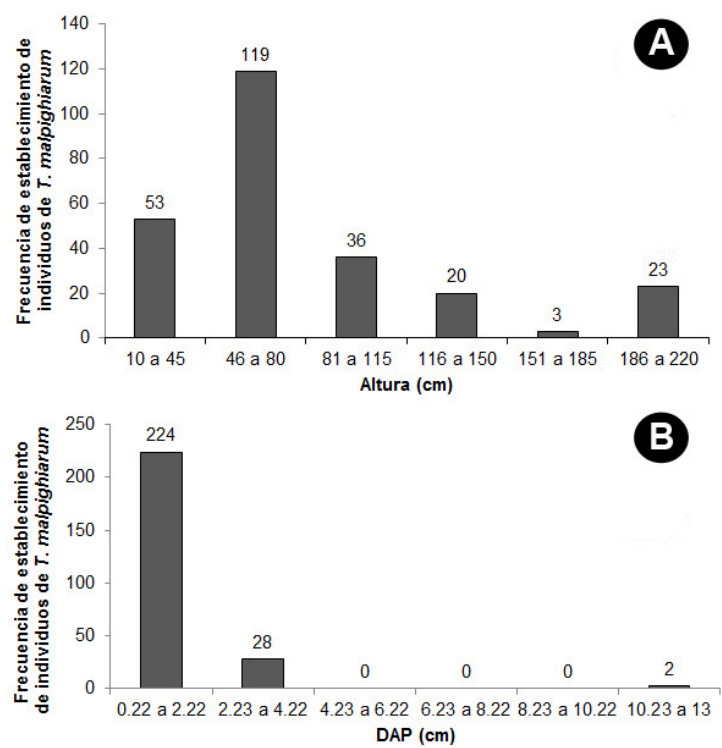

Figura 3. Frecuencia de establecimiento de individuos de

Tetramicra malpighiarum (Orchidaceae) en ramas a distintas alturas (A) y grosores (B), en el Parque Nacional Desembarco del Granma, Cuba.

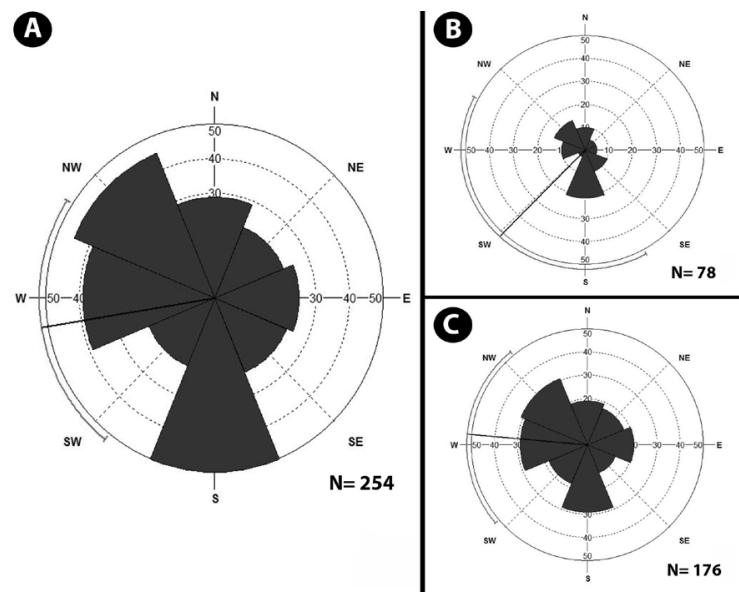

Figura 4. Diagramas de rosa de las orientaciones cardinales de Tetramicra malpighiarum (Orchidaceae), en el Parque Nacional Desembarco del Granma, Cuba. A: Población total; B: Individuos inmaduros; C: Individuos adultos. Los pétalos más largos representan las orientaciones más frecuentes. Intervalos de confianza del $95 \%$.

cardinales, (Fig. 4A), aunque existen diferencias significativas entre ellas (Uniformidad Circular de Rayleigh, $\mathrm{z}=3.543, \mathrm{p}=0.029)$. La población muestra una tendencia a crecer preferentemente con orientaciones sur (19.68\% del total de individuos; 50 individuos) y noroeste (17.72\% del total de individuos; 
45 individuos), concentrándose en estas direcciones $37.4 \%$ de los individuos (95 individuos; Fig. 4A). Esta tendencia numérica también se mantiene cuando se analiza aisladamente el número de individuos inmaduros (sur: $26.92 \%$ del total de individuos en esta clase de vida; noroeste: $17.95 \%$; en conjunto: $44.9 \%$; Fig. 4B), aunque no se aprecian diferencias estadísticamente significativas (Uniformidad Circular de Rayleigh, $z=1.172, p=0.31)$. En cuanto a los individuos adultos, también se mantiene la tendencia general (sur: $16.48 \%$ del total de individuos en esta clase de vida; noroeste: $17.61 \%$; en conjunto: $34.09 \%$; Fig. 4C), pero en este estadio en el número de individuos hacia las diferentes orientaciones si es estadísticamente significativo (Uniformidad Circular de Rayleigh, $\mathrm{z}=3.036, \mathrm{p}=0.048$ ).

Discusión. A pesar de que en la población estudiada, el número de individuos de T. malpighiarum continúa siendo bajo y se concentran en una área muy reducida, este número de efectivos poblacionales supera en $1.69 \%$ al número aproximado de individuos (aproximadamente 150 individuos) encontrado por García-González et al. (2013), en un estudio preliminar desarrollado en este mismo sitio. El mayor número de individuos adultos en la población puede ser indicativo de una población antigua (Chung et al. 2011), y estos individuos pueden influir sustancialmente en la reproducción y expansión de la especie, al ser la etapa adulta la más importante en la vida de las orquídeas (Zotz 1998, Winkler \& Hietz 2001, Mondragón 2009). Sin embargo, el bajo número de individuos inmaduros puede indicar una población relativamente "regresiva" o "senil" (Hutchings et al. 1998), lo cual, unido a su bajo número de efectivos poblacionales de forma general, haría vulnerable a la especie.

No obstante, es importante tener en cuenta, que en el caso de algunas especies de orquídeas, el número de individuos en cada estadio de vida puede ser dinámico a lo largo de los años. Pueden predominar los individuos inmaduros o adultos indistintamente, dependiendo de ciclos naturales de las poblaciones o determinado por factores externos, como el clima (fundamentalmente temperatura y humedad) y modificaciones naturales y/o antrópicas en el hábitat donde se desarrollan, modificaciones que pueden ser tanto perjudiciales como beneficiosas (Larson 1992, Hutchings et al.
1998, Olaya-Arenas et al. 2011, Mújica et al. 2013).

En la población de T. malpighiarum fueron mínimas las diferencias en el número de individuos creciendo agregados y creciendo solitarios, y este parámetro es independiente del estadio de vida de los individuos. Sin embargo, el crecimiento agregado es un fenómeno muy común en muchas especies de orquídeas epífitas (Ackerman 1995, Hietz \& Hietz-Seifert 1995, Tremblay 1997). Por ejemplo, frecuentemente I. utricularioides y B. cubensis crecen en parches o en agregación, siendo ellas mismas las especies con las que más comúnmente se agregan (González et al. 2007, Raventós et al. 2011, GarcíaGonzález \& Riverón-Giró 2014).

Aunque se plantea que las epifitas vasculares generalmente presentan bajos niveles de competencia intraespecífica (Zotz \& Hietz 2001), lo que podría explicar que los individuos de T. malpighiarum crezcan indistintamente solitarios o en agregación, esta afirmación es contradictoria. Aunque las semillas de la mayoría de las especies (incluyendo T. malpighiarum) se dispersan por el aire y pueden recorrer largas distancias (Arditti \& Abdul Ghani 2000, Hágsater et al. 2005), tienen más probabilidades de caer cerca de la planta madre (Machon et al. 2003, Trapnell et al. 2004, Jersáková \& Malinová 2007, Winkler et al. 2009, Chung et al. 2011), lo que puede representar una ventaja, al tener mayores posibilidades de que encuentren los hongos micorrícicos que necesitan para germinar (Batty et al. 2001, Diez 2007).

En general, los patrones de dispersión de las semillas de orquídeas son determinados por la distribución espacial de los adultos, su lluvia de semillas y su sombra de semillas, mientras que el reclutamiento de plántulas depende principalmente de la probabilidad de llegada de las semilla y la disponibilidad de un micrositio adecuado (Nathan \& Muller-Landau 2000). Estudios de varias especies de orquídeas terrestres han demostrado que muchas semillas caen a una distancia muy corta de la planta de origen (entre $15 \mathrm{~cm}$ y $7 \mathrm{~m}$; Machon et al. 2003, Jacquemyn et al. 2007, Jersáková \& Malinová 2007).

Es importante determinar la distancia promedio de caída de semillas de T. malpighiarum y comprobar con estudios a largo plazo, si la distancia de agregación considerada por los autores $(\leq 5 \mathrm{~cm})$ es adecuada. A pesar de que los individuos de T. malpighiarum crezcan 
o no agregados entre ellos o con otras especies de epífitas vasculares, su raíces son capaces de extenderse a mayores longitudes, pudiendo entrelazarse con las raíces de otras orquídeas de su misma especie o de otras epífitas vasculares, pudiendo establecer interacciones que probablemente puedan tener implicaciones positivas o negativas para los individuos. Por ejemplo, García-González \& Riverón-Giró (2014), refieren que en el caso de I. utricularioides, los individuos que crecen en ramillas, agregados con otras plantas de su misma especie o con otras especies de epífitas vasculares, pueden tener mayores ventajas competitivas y mayores posibilidades de supervivencia. Sin embargo, en $T$. malpighiarum, al ser una especie mucho más pequeña, no se pudo observar tan claramente estos posibles beneficios.

Tetramicra malpighiarum manifiesta una fuerte tendencia a preferir como forófito a $M$. incana, una especie endémica de las Grandes Antillas, nativa de Cuba y Jamaica (Acevedo-Rodríguez \& Strong 2012). Su nivel de ocupación es mucho mayor que el de las demás especies arbustivas y arbóreas del área, solo seis forófitos fueron de otras especies $(30 \%$ del total de forófitos). Esta preferencia de T. malpighiarum ya había sido reportada anteriormente por Hernández \& Díaz (2000) y Llamacho \& Larramendi (2005), que la habían encontrado creciendo exclusivamente sobre M. incana, aunque García-González et al. (2013) localizaron dos individuos creciendo también sobre Erythroxylum havanense Jacq. (Erythroxylaceae). En este estudio se reporta por primera vez a $T$. malpighiarum ocupando otras cinco especies de forófitos (además de $M$. incana y E. havanense), lo cual reafirma la rareza de la especificidad de hospedero (Benzing 1990) y la tendencia entre las epífitas a ocupar más de una especie de árbol o arbusto (Martínez-Meléndez et al. 2008).

Hipotéticamente se esperaría que las epífitas puedan establecerse en cualquier hospedero indistintamente (Callaway et al. 2002), no obstante, los estudios sobre este aspecto son pocos y no concluyentes (Trapnell \& Hamrick 2006). Algunos autores han encontrado baja especificidad entre las epífitas y los forófitos que ocupan (Johansson 1974, Ackerman et al. 1989, Zimmerman \& Olmsted 1992, Ackerman et al. 1996), mientras otros han encontrado una fuerte asociación (Went 1940, Frei 1973, Ter Steege \& Cornelissen
1989, Tremblay et al. 1998, Cedillo et al. 2013).

En estudios desarrollados con Laelia rubescens Lindl., se encontró asociación entre esta especie y Samanea saman (Jacq.) Merr. (Fabaceae), aunque la orquídea puede crecer sobre otros forófitos (Trapnell \& Hamrick 2006). En otro trabajo desarrollado con Dendrophylax lindenii (Lindl.) Benth. ex Rolfe, los autores encontraron que esta especie muestra una estrategia generalista en la selección del sustrato, pudiendo encontrarse creciendo en 19 especies de forófitos, aunque manifiesta una clara tendencia a preferir tres de ellos (Mújica et al. 2010). Igualmente la comunidad de forófitos ocupados por B. cubensis es diversa, con 14 especies, pero se observa una preferencia por dos de ellas (González et al. 2007). Sin embargo, la especificidad estricta de una especie de orquídea epífita con una especie de forófito es un fenómeno poco común (Tremblay et al. 1998). Por ejemplo, se observó que Quercus desertícola Trel. (Fagaceae) es el hospedero específico de Prosthechea aff. karwinskii (Martius) Soto-Arenas \& Salazar (Cedillo et al. 2013), Quercus elliptica Née (Fagaceae) parece ser el único hospedero de Hagsatera brachycolumna (L.O. Williams) R. González (Hágsater et al. 2005), y Tremblay et al. (1998) encontraron que Lepanthes caritensis Tremblay \& Ackerman solamente coloniza forófitos de la especie Micropholis guyanensis (A. DC.) Pierre (Sapotaceae).

Teniendo en cuenta que en el área de estudio las especies de forófitos crecen entremezcladas, se localizan principalmente en el sotobosque y generalmente presentan porte y arquitectura similares (excepto Plumeria obtusa y Guettarda elliptica, que son árboles pequeños, que puede alcanzar hasta 7.6 m y 6 m de altura respectivamente; Francis 2004, MBG 2015b), la presencia de T. malpighiarum sobre estas especies, fundamentalmente sobre $M$. incana, posiblemente estén asociadas a características particulares de estos taxones. Algunas de estas características pueden ser, el tamaño del hospedero, la estructura de copa poco densa y con abundancia de ramas finas, la textura y composición química de la corteza (Ter Steege \& Cornelissen 1989, Hietz \& Hietz-Seifert 1995, Callaway et al. 2002, González et al. 2007, Krömer et al. 2007), y la presencia probable del/los hongo/s micorrícico/s con los que necesariamente debe asociarse T. malpighiarum para 
germinar y desarrollarse (Otero et al. 2004, Trapnell \& Hamrick 2006, Jersáková \& Malinová 2007, Mújica 2007, Otero et al. 2007, Mújica et al. 2010, Chung et al. 2011).

Otro factor a tener en cuenta en la relación de $T$. malpighiarum con sus forófitos es lo planteado por Llamacho \& Larramendi (2005), quienes mencionan que en $M$. incana, la orquídea se asocia con un liquen que crece en la corteza de esta especie, aunque no aclaran la identidad del liquen. Es fundamental realizar estudios que corroboren esta relación, que permitan identificar la especie de liquen y que permitan conocer si este taxón está presente también en las demás especies de forófito hacia las que se ha diversificado $T$. malpighiarum, facilitando su establecimiento en estos sustratos.

A pesar de que se requiere un estudio específico para poder descartar el posible efecto de un sesgo en la disponibilidad de ramas en los forófitos, y entonces poder hablar de preferencias, se puede inferir que existen ciertas tendencias de establecimiento en los individuos de T. malpighiarum. Más de $90 \%$ de la población de T. malpighiarum crecía en las ramillas, distribución que probablemente esté relacionada con lo planteado por Johansson (1974) y Krömer et al. (2007), cuando afirman que las epífitas vasculares tienden a mostrar patrones de distribución vertical en sus forófitos que reflejan su rango de tolerancia a la luz, la humedad y otras adaptaciones ecofisiológicas. Las ramillas es el microhábitat más expuesto del forófito, con abundancia de ramas finas sometidas más directamente a las corrientes de aire, a mayor intensidad luminosa, a mayor desecación y con poca disponibilidad de nutrientes (Hágsater et al. 2005). Tetramicra malpighiarum probablemente encuentra en este microhábitat las condiciones más adecuadas para su desarrollo, presentando adaptaciones que facilitan su desarrollo y permanencia en este ambiente. Son plantas sin pseudobulbos, ligeras y pequeñas, de hasta $6 \mathrm{~cm}$ de alto, tienen hojas (entre 3-7) erectas o falciformes, cilíndricas, suculentas y de cutículas gruesas, que le permiten acumular y conservar agua en sus tejidos, hojas dispuestas en forma de roseta, y raíces delgadas $(0.5-1 \mathrm{~mm}$ de diámetro) y numerosas (Hernández \& Díaz 2000, Llamacho \& Larramendi 2005, Ackerman 2014b), características todas típicas de las llamadas orquídeas de ramilla (Hágsater et al. 2005). De la biología de este tipo de epífitas aún se desconocen muchos aspectos, planteándose por ejemplo que florecen a muy temprana edad (unos meses o un año después de germinar) y que tienen tiempos generacionales muy cortos (de 1-5 años; Hágsater et al. 2005), por lo que se hace imprescindible sistematizar los estudios de $T$. malpighiarum para comprobar estos aspectos de gran relevancia para su conservación.

A pesar de que T. malpighiarum se localizó creciendo hacia las ocho orientaciones cardinales, no se encontró un factor específico que permitiera explicar la tendencia contrastante que presentaron los individuos, de ubicarse preferentemente hacia el sur y el noroeste. Este comportamiento poblacional puede ser aleatorio, consecuencia de un factor especifico que es necesario probar, o resultado de una combinación de factores como, la dirección y velocidad predominante de los vientos del área (González et al. 2007, Tremblay \& Velázquez 2009), las necesidades particulares de luz y humedad de esta orquídea, y/o de la orientación de los sitios donde se encuentran las plantas en los forófitos, en relación con los demás árboles o arbustos que los rodean (Mújica 2007). En Puerto Rico, Tremblay \& Velázquez (2009), encontraron que Lepanthes eltoroensis Stimson también manifiesta preferencia por crecer en el lado noroeste de los troncos de los árboles que ocupa, probablemente a consecuencia de los constantes vientos que suben del mar Caribe, principalmente vientos del este y del noreste. Sin embargo, para Ionopsis utricularioides (Sw.) Lindl., García-González \& Riverón-Giró (2014), plantean que probablemente la orientación cardinal de las plantas es completamente aleatoria, siendo esta especie más susceptible a factores como la luz y el grosor de la rama. En el PNDG, los vientos son persistentes durante todo el año, predominantemente del este-sureste al sureste (excepto en temporada invernal, donde se presentan vientos del norte), y a ellos se asocian las mayores velocidades (Palacio et al. 2012). En El Guafe, con respecto al mar, la población de T. malpighiarum se localiza aproximadamente a 2.5 $\mathrm{km}$ en línea recta al sur, a $2.9 \mathrm{~km}$ en dirección sureste, a $3.7 \mathrm{~km}$ en dirección noroeste, y a $6.5 \mathrm{~km}$ en dirección norte, por lo que estos vientos probablemente sean una fuente importante de humedad para la especie durante todo el año. 
Es fundamental continuar monitoreando esta especie y desarrollar estudios demográficos a largo plazo, para lograr obtener datos cuantitativos relativos a su tasa de floración, polinización, germinación y mortalidad en el PNDG; datos que permitirán apreciar exactamente el estado actual de la población y su viabilidad. También, es imprescindible emprender estudios similares en la otra población conocida de la especie, al centro de la isla (Hernández \& Díaz 2000, Llamacho \& Larramendi 2005), vinculados con estudios moleculares, que permitan conocer los niveles de endogamia y variabilidad genética de los individuos. Estos aspectos pueden tener grandes implicaciones en la conservación de la especie (Chung et al. 2011), más considerando que ambas poblaciones están aisladas y son muy reducidas (Hernández \& Díaz 2000, Llamacho \& Larramendi 2005, Ackerman 2014b).

Agradecimientos. A los trabajadores del Parque Nacional Desembarco del Granma, por el apoyo brindado. A José Luis Gámez y Eldis Bequer por su gran ayuda en la identificación de las especies de forófitos. A la organización IDEA WILD por el equipamiento proporcionado, fundamental para la realización de este trabajo.

\section{LiTEARATURA CITADA}

Acevedo-Rodríguez, P. \& Strong, M. T. (2012). Catalogue of seed plants of the West Indies. Smithsonian Contributions to Botany. Number 98. Smithsonian Institution Scholarly Press. Washington D.C., E.U.

Ackerman, J. D. (1995). An orchid flora of Puerto Rico and the Virgin Islands. Memoir of the New York Botanical Garden, vol. 73, The New York Botanical Garden Press, Nueva York, E.U.

Ackerman, J. D. (2014a). Orchidaceae A. Jussieu. En: J. D. Ackerman (Coor.), Orchid flora of the Greater Antilles (pp. 7-8). Memoirs of the New York Botanical Garden, vol. 109, The New York Botanical Garden Press, Nueva York, E.U.

Ackerman, J. D. (2014b). Tetramicra. En: J. D. Ackerman (Coor), Orchid flora of the Greater Antilles (pp. 497506). Memoirs of the New York Botanical Garden, vol. 109, The New York Botanical Garden Press, Nueva York, E.U.

Ackerman, J. D., Montalvo, A. M. \& Vera, A. M. (1989). Epiphyte host specifity of Encyclia krugii, a Puerto Rican endemic orchid. Lindleyana, 4(2), 74-77.

Ackerman, J. D., Sabat, A. \& Zimmerman, J. K. (1996). Seedling establishment in an epiphytic orchid: an experimental study of seed limitation. Oecologia, 106, 192-198.

Arditti, J. \& Abdul Ghani A.K. (2000). Tansley Review No. 110. Numerical and physical properties of orchid seeds and their biological implications. New Phytologist, 145, 367-421. DOI: $10.1046 / j .1469-8137.2000 .00587 . x$

Batty, A. L., Dixon, K. W., Brundrett, M. \& Sivasithamparam, K. (2001). Constraints to symbiotic germination of terrestrial orchid seed in a mediterranean bushland. New Phytologist, 152, 511-520. DOI: 10.1046/j.0028646X.2001.00277.x

Benzing, D. H. (1990). Vascular epiphytes. Cambridge University, Nueva York, E.U.

Berazaín, R., Areces, F., Lazcano, J. C. \& González-Torres, L. R. (2005). Lista roja de la flora vascular cubana. Documentos del Jardín Botánico Atlántico, Volumen 4. Gijón, España.

Callaway, R. M., Reinhart, K. O., Moore, G. W., Moore, D. J. \& Pennings, S. C. (2002). Epiphyte host preferences and host traits: mechanisms for species specific interactions. Oecología, 132, 221-230. DOI: 10.1007/ s00442-002-0943-3

Cedillo, L. L., Gómez-Alonso, C. \& Ávila-Díaz, I. (2013). Patrones de distribución vertical y horizontal de la epífita endémica Prosthechea aff. karwinskii (Orchidaceae) en Michoacán, México. Lankesteriana, 13(1-2), 140.

Chung, M. Y., Nason, J. D. \& Chung, M. G. (2011). Significant demographic and fine-scale genetic structure in expanding and senescing populations of the terrestrial orchid Cymbidium goeringii (Orchidaceae). American Journal of Botany, 98(12), 2027-2039. DOI: 10.3732/ajb.1100094

Díaz, M. A. (1999). Orquídeas cubanas: Pasado, presente y futuro. Seminario Mesoamericano de Orquideología y Conservación. Universidad de Costa Rica, San José, Costa Rica.

Diez, J. M. (2007). Hierarchical patterns of symbiotic orchid germination linked to adult proximity and environmental gradients. Journal of Ecology, 95, 159170. DOI: 10.1111/j.1365-2745.2006.01194.x

Francis, J. K. (2004). Guettarda elliptica Sw. En: J. K. Francis (Ed.), Wildland Shrubs of the United States and its territories: Thamnic descriptions, Volume 1. (pp. 363-364). General Technical Report IITF-GTR-26. United States Department of Agriculture (USDA), Forest Service, E.U., International Institute of Tropical Forestry, San Juan, Puerto Rico y Rocky Mountain Research Station, E.U.

Frei, S. J. K. (1973). Orchid ecology in a cloud forest in the mountains of Oaxaca, Mexico. American Orchid Society Bulletin, 42, 307-314.

García-González, A., Damon, A., Esparza, L. G. \& ValleMora, J. (2011). Population structure of Oncidium 
poikilostalix (Orchidaceae), in coffee plantations in Soconusco, Chiapas, México. Lankesteriana, 11(1), 23-32.

García-González, A., Riverón-Giró, F. B. \& Mújica, E. (2013). Tetramicra malpighiarum J.A. Hern. \& M.A. Díaz. En: L. R. González-Torres, A. Palmarola, D. Barrios \& L. González-Oliva (Eds.), Top 50: Las 50 plantas más amenazadas de Cuba (pp. 100-101). Bissea 7(número especial), 1.

García-González, A. \& Riverón-Giró, F. B. (2014). Organización espacial y estructura de una población de Ionopsis utricularioides (Orchidaceae) en un área suburbana de Pinar del Río, Cuba. Lankesteriana, 13(3), 419-427.

García-González, A. \& Pérez, R. (2011). La comunidad orquideológica en la Reserva de la Biosfera Sierra del Rosario, Cuba. Revista de Biología Tropical, 59(4), 1805-1812.

González, E., Raventós, J., Mújica, E. \& Bonet, A. (2007). Estructura y ecología de la población del endemismo cubano Broughtonia cubensis (Orchidaceae), en Cabo San Antonio, Península de Guanahacabibes, provincia de Pinar del Río, Cuba. Lankesteriana, 7(3), 469-478.

González-Torres, L. R., Palmarola, A., Bécquer, E. R., Berazaín, R., Barrios, D. \& Gómez, J. L. (2013). Las 50 plantas más amenazadas de Cuba. En: L. R. GonzálezTorres, A. Palmarola, D. Barrios \& L. González-Oliva (Eds.), Top 50: Las 50 plantas más amenazadas de Cuba (pp. 4-5). Bissea 7(número especial), 1.

Hágsater, E., Soto, M. A., Salazar, G. A., Jiménez, R., López, M. \& Dressler R. L. (2005). Las orquídeas de México. Instituto Chinoín. México D.F., México.

Hernández, J. A. \& Díaz, M. A. (2000). A new species of Tetramicra (Orchidaceae) from eastern Cuba. Harvard Papers in Botany, 5(1), 189-192.

Hietz, P. \& Hietz-Seifert, U. (1995). Intra and interspecific relations within an epiphyte community in a Mexican humid montane forest. Selbyana, 16, 135-140.

Hutchings, M. J., Mendoza, A. \& Havers, W. (1998). Demographic properties of an outlier population of Orchis militaris L. (Orchidaceae) in England. Botanical Journal of the Linnean Society, 126(1ロ2), 95-107. DOI: 10.1111/j.1095-8339.1998.tb02519.x

Jacquemyn, H., Brys, R., Vandepitte, K., Honnary, O., Roldán-Ruiz, I. \& Wiegand, T. (2007). A spatially explicit analysis of seedling recruitment in the terrestrial orchid Orchis purpurea. New Phytologist, 176, 448459. DOI: $10.1111 /$ j.1469-8137.2007.02179.x

Jersáková, J. \& Malinová, T. (2007). Spatial aspects of seed dispersal and seedling recruitment in orchids. New Phytologist, 176, 237-241. DOI: 10.1111/j.14698137.2007.02223.x
Johansson, D. (1974). Ecology of vascular epiphytes in West African rain forest. Acta Phytogeographica Suecica, 59, 1-129.

Krömer, T., Kessler, M. \& Gradstein, S. R. (2007). Vertical stratification of vascular epiphytes in submontane and montane forest of the Bolivian Andes: the importance of the understory. Plant Ecology, 189(2), 261-278.

Larson, R. J. (1992). Population dynamics of Encyclia tampensis in Florida. Selbyana, 13, 50-56.

Llamacho, J. A. \& Larramendi, J. A. (2005). Las orquídeas de Cuba. Greta, Sevilla, España.

Machon, N., Bardin, P., Mazer, S. J., Moret, J., Godelle, B. \& Austerlitz, F. (2003). Relationship between genetic structure and seed and pollen dispersal in the endangered orchid Spiranthes spiralis. New Phytologist, 157, 677687. DOI: 10.1046/j.1469-8137.2003.00694.x

Martínez-Meléndez, N., Pérez-Farrera, M. A. \& FloresPalacios, A. (2008). Estratificación vertical y preferencia de hospedero de las epífitas vasculares de un bosque nublado de Chiapas, México. Revista de Biología Tropical, 56(4), 2069-2086. DOI: 10.15517/ rbt.v56i4.5780

MBG. (2015a). Tropicos. Missouri Botanical Garden. (Consultado: 20 de junio de 2015, http://www.tropicos. org/).

MBG. (2015b). Plumeria obtusa. Plant Finder. Missouri Botanical Garden. (Consultado: 20 de junio de 2015, http://www.missouribotanicalgarden.org/PlantFinder/ PlantFinderDetails.aspx?kempercode $=\mathrm{f} 412$ ).

Mondragón, D. (2009). Population viability analysis for Guarianthe aurantiaca, an ornamental epiphytic orchid harvested in Southeast México. Plant Species Biology, 24, 35-41. DOI: 10.1111/j.1442-1984.2009.00230.x

Mújica, E. (2007). Ecología de las orquídeas epífitas Broughtonia cubensis (Lindley) Cogniaux, Dendrophylax lindenii (Lindley) Bentham et Rolfe y Encyclia bocourtii Mújica et Pupulin en el Cabo San Antonio, Península de Guanahacabibes, Cuba. Análisis espacio-temporal e implicaciones del impacto de un fenómeno atmosférico severo. Tesis en opción al grado científico de Doctor en Ciencias. Universidad de Alicante, España.

Mújica, E., Raventós, J., González, E. \& Bonet, A. (2013). Long-term hurricane effects on populations of two epiphytic orchid species from Guanahacabibes Peninsula, Cuba. Lankesteriana, 13(1-2), 47-55.

Mújica, E., Raventós, J. \& González, E. (2010). Análisis de la selección de sustrato por parte de Dendrophylax lindenii (Orchidaceae) en Cabo San Antonio, Península de Guanahacabibes, Pinar del Río, Cuba. Lankesteriana, 9(3), 533-540.

Nathan, R. \& Muller-Landau, H. C. (2000). Spatial patterns of seed dispersal, their determinants and consequences 
for recruitment. Trends in Ecology and Evolution, 15(7), 278-285.

Olaya-Arenas, P., Meléndez-Ackerman, E. J., Eglee, M. \& Tremblay, R. (2011). Demographic response by a small epiphytic orchid. American Journal of Botany, 98(12), 2040-2048. DOI: 10.3732/ajb.1100223

Otero, J. T., Ackerman, J. D. \& Bayman, P. (2004). Differences in mycorrhizal preferences between two tropical orchids. Molecular Ecology, 13, 2393-2404. DOI: 10.1111/j.1365-294X.2004.02223.x

Otero, J. T., Flanagan, N. S., Herre, E. A., Ackerman, J. D. \& Bayman, P. (2007). Widespread mycorrhizal specificity correlates to mycorrhizal function in the neotropical, epiphytic orchid Ionopsis utricularioides (Orchidaceae). American Journal of Botany, 94(12), 1944-1950. DOI: 10.3732/ajb.94.12.1944

Palacio, E., Escalona, R., Cala, Y. R., Calaña, S., Ocano, C. A., Alayón, L., Pérez, J., Cisneros, G. \& Sariego, O. (2012). Plan de Manejo Parque Nacional Desembarco del Granma (2012-2016). Ministerio de la Agricultura (MINAGRI), Cuba. Empresa Nacional para la Protección de la Flora y la Fauna (ENPFF), Cuba.

Raventós, J., Mújica, E., Wiegand, T. \& Bonet, A. (2011). Analyzing the spatial structure of Broughtonia cubensis (Orchidaceae) populations in the dry forests of Guanahacabibes, Cuba. Biotropica, 43(2), 173-182. DOI: $10.1111 / \mathrm{j} .1744-7429.2010 .00684 . x$

Ter Steege, H. \& Cornelissen, J. H. C. (1989). Distribution and Ecology of vascular epiphytes in lowland rain forest of Guyana. Biotropica, 21(4), 331-339. DOI: $10.2307 / 2388283$

Trapnell, D. W., Hamrick J. L. \& Nason, J. D. (2004). Three-dimensional fine-scale genetic structure of the neotropical epiphytic orchid Laelia rubescens. Molecular Ecology, 13, 1111-1118. DOI: 10.1111/j.1365-294X.2004.02148.x

Trapnell, D. W. \& Hamrick, J. L. (2006). Variety of Phorophyte species colonized by the neotropical epiphyte, Laelia rubescens (Orchidaceae). Selbyana, 27, 60-64.

Tremblay, R. L. (1997). Distribution and dispersion patterns of individuals of nine species of Lepanthes (Orchidaceae). Biotropica, 29, 38-45. DOI: 10.1111/ j.1744-7429.1997.tb00004.x

Tremblay, R. L., Zimmerman, J. K., Lebrón, L., Bayman, P., Sastre, I., Axelrod, K. \& Alers-García, J. (1998). Host specifity and low reproductive succee in the rare endemic Puerto Rican orchid Lepanthes caritensis. Biological Conservation, 85(3), 297-304. DOI: 10.1016/S0006-3207(97)00163-8

Tremblay, R. L. \& Velázquez, J. (2009). Circular distribution of an epiphytic herb on trees in subtropical rain forest. Tropical Ecology, 50(2), 211-217.

Went, F. W. (1940). Soziologie der Epiphyten eines tropishen Urwaldes. Ann Jard Bot Buitenzorg, 50, 1-98.

Whittaker, R. J. \& Fernández-Palacios, J. M. (2007). Island biogeography. Ecology, evolution, and conservation. Oxford University Press, Oxford, E.U.

Winkler, M. \& Hietz, P. (2001). Population structure of three epiphytic orchids (Lycaste aromatica, Jacquiniella leucomelana, and $J$. teretifolia) in a Mexican humid montane forest. Selbyana, 22, 27-33.

Winkler, M., Hülber, K. \& Hietz, P. (2009). Population dynamics of epiphytic orchids in a metapopulation context. Annals of Botany, 104(5), 995-1004. DOI: 10.1093/aob/mcp188

Zimmerman, J. K. \& Olmsted, I. C. (1992). Host tree utilization by vascular epiphytes in a seasonally inundated forest (tintal) in Mexico. Biotropica, 24(3), 402-407. DOI: 10.2307/2388610

Zotz, G. (1998). Demography of the epiphytic orchid, Dimerandra emarginata. Journal of Tropical Ecology, 14(06), 725-741. DOI: 10.1017/S0266467498000534

Zotz, G. \& Hietz, P. (2001). The ecophysiology of vascular epiphytes: current knowledge, open questions. Journal of Experimental Botany, 52(364), 2067-2078. 
